\title{
Monte Carlo Study on Number of Scattering Events for Quasi- Ballistic Transport in MOSFETs
}

\author{
Y. Ohkura and C. Suzuki \\ Semiconductor Leading Edge Technologies, Inc. Tsukuba, Ibaraki, JAPAN \\ ohkura.yasuyuki@selete.co.jp
}

\begin{abstract}
Role of scattering has been discussed as scattering still controls drain current of decananometer MOSFET's, including the number of scattering events per unit length or gate length. Nevertheless, as scattering mechanisms have various angular dependences, in this paper, meanings of 'number' of scattering events are discussed and 'effective' scattering number is introduced to interpret quasi-ballistic transport. This concept is shown to be useful to understand the quasi ballistic transport and the role of various scattering mechanisms especially when back-scattering is not negligible.
\end{abstract}

\section{Introduction}

There have been many discussions on quasi-ballistic transport since Ref. [1] [2]. Role of scattering has been discussed as scattering still controls drain current of decananometer MOSFET's, including the number of scattering events per unit length [3] or gate length [4]. Nevertheless, as scattering mechanisms have various angular dependences, in this paper, meanings of 'number' of scattering events are discussed and 'effective' scattering number is introduced to interpret quasi-ballistic transport.

\section{Simulation results and discussion}

The calculate example is a typical bulk n-MOSFET with $\mathrm{L}_{\mathrm{g}}=45 \mathrm{~nm}, \mathrm{~T}_{\mathrm{ox}}=1.2 \mathrm{~nm}$ presented elsewhere [5], using in-house self-consistent fullband Monte Carlo simulator, HyDeLEOSMC [6].

In this structure, cumulative average numbers of scattering events at $\mathrm{V}_{\mathrm{d}}=\mathrm{V}_{\mathrm{g}}=0.9 \mathrm{~V}$ $\left(I_{d}=1.1 \mathrm{~mA} / \mu \mathrm{m}\right)$, counted along the channel starting from the source junction point are shown in Fig. 1, with and without carrier-carrier scattering (CCS). Numbers of phonons scattering (phonon), surface scattering (surf), carrier-carrier scatterings and their total (total) are shown. About 3 million events are counted for 50 thousand Monte Carlo particles. The number of surface scattering is dominant and that of CCS is relatively large. Surface scattering is modelled from specular-diffusive approach [7] (with $p=0.2$ ). And CCS is modelled from Ref. [8], which has been known to be anisotropic. Thus, the scattering number is not proper indicator, so the concept of the number of scatterings is investigated in this paper.

At a scattering event, the carrier velocity is modified, so the velocity modification is added up as the 'cumulative loss of velocity' and shown in Fig. 2. The total values at 
the drain junction are up to $210^{8} \mathrm{~cm} / \mathrm{s}$, twenty times of so-called saturation velocity. The contribution of CCS is shown to be relatively small, thus the "cumulative loss of velocity' is thought to be one of valuable indicators to discuss ballistic transport.

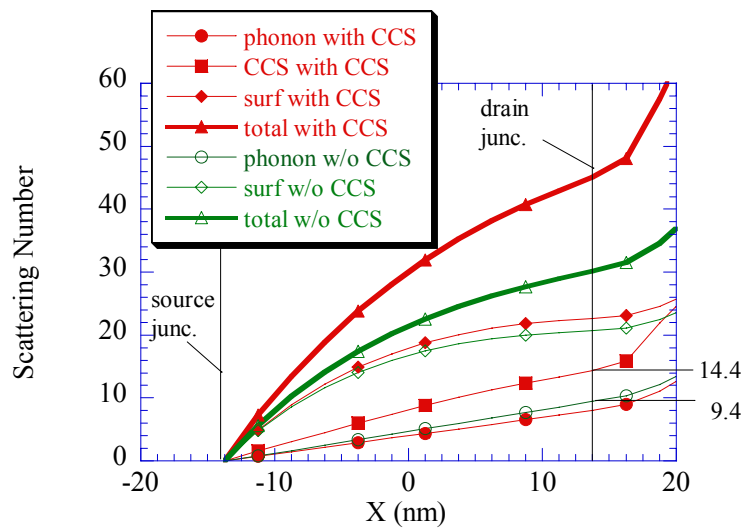

Figure 1: Cumulative numbers of scattering events along the channel starting from the source junction point with / without (w/o) carrier-carrier scatterings (CCS) for a typical n-MOSFET with $\mathrm{L}_{\mathrm{g}}=45 \mathrm{~nm}, \mathrm{~L}_{\text {met }}=27.5 \mathrm{~nm}, \mathrm{~T}_{\mathrm{ox}}=1.2 \mathrm{~nm}, \mathrm{~V}_{\mathrm{d}}=\mathrm{V}_{\mathrm{g}}=0.9 \mathrm{~V}$ and $\mathrm{T}=300 \mathrm{~K}$. Numbers of phonons scattering (phonon), surface scattering (surf), carrier-carrier scatterings (CCS) and their total (total) are shown.

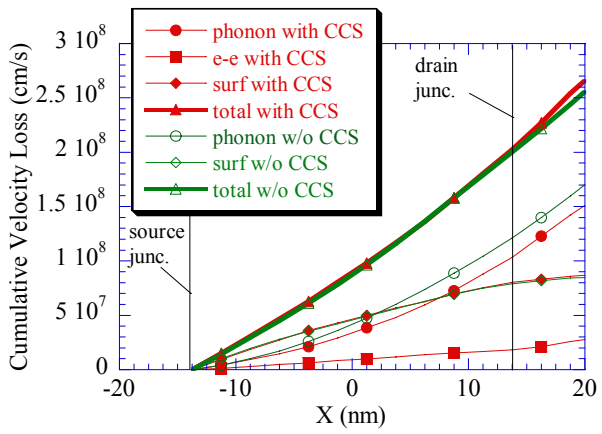

Figure 2: Cumulative velocity losses along the channel starting from the source junction point.

Nevertheless, the 'cumulative loss of velocity' might not be familiar, so we propose the 'effective' scattering number, defined by the division of the velocity loss by the root of the mean square velocity of $\mathrm{x}$-direction, $\sqrt{\left\langle V_{x}^{2}\right\rangle}$. The cumulative results are shown in Fig. 3. The effective scattering number of CCS is 0.69 at the drain junction, only $4.8 \%$ of corresponding scattering number (14.4), and this relation can be ascribed to the anisotropic nature of CCS. The effective scattering number of phonons at the drain junction is 4.0 (w/o CCS), $42 \%$ of corresponding scattering number (9.4). 


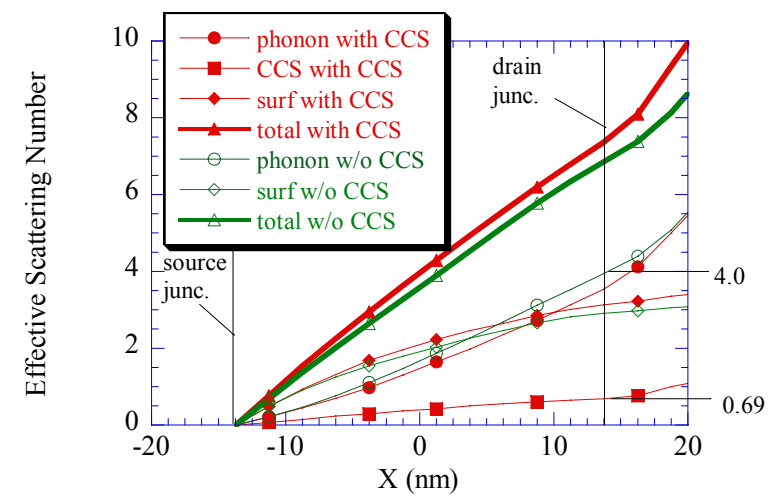

Figure 3: Cumulative effective numbers of scattering events counted along the channel starting from the source junction point.

This difference between scattering number and effective scattering number of phonons is attributed to large ratio of back-scattering. In Fig. 4, back-scattering coefficients (BSC) along the channel are shown, and their definitions are described in the figure caption. The values of BSC are large compared to the reported results in DGSOIFET [4]. The ratio of effective scattering number to scattering number of phonons is approximately related to a factor $F$ derived from BSC as,

$$
F=\frac{\left|J_{f w d}\right|-\left|J_{b w d}\right|}{\left|J_{f w d}+J_{b w d}\right|}=\frac{1-\left|J_{b w d} / J_{f w d}\right|}{1+\left|J_{b w d} / J_{f w d}\right|}
$$

The BSC, $\left|J_{b w d} / J_{f w d}\right|$, throughout channel region is about 0.4 in average, thus $F$ is about $42 \%$, which is thought to be in good correspondence.

In Fig. 4, BSCs at $\mathrm{V}_{\mathrm{g}}=0.9 \mathrm{~V}$ are larger than that at $\mathrm{V}_{\mathrm{g}}=0.3 \mathrm{~V}(\mathrm{Id}=0.21 \mathrm{~mA} / \mu \mathrm{m})$ in the channel near the drain junction but the relation is reversed at near the source junction. For explanation, the distribution of potential is shown in Fig. 5, as BSC is usually discussed at the 'potential minima' $\left(\psi_{\min }\right)$. The asterisks $(*)$ indicate the position of $\psi_{\min }$, which moves to the source side with increase of $\mathrm{V}_{\mathrm{g}}$, thus BSC at $\psi_{\text {min }}$ is smaller at $\mathrm{V}_{\mathrm{g}}=0.3 \mathrm{~V}$ than that at $\mathrm{V}_{\mathrm{g}}=0.9 \mathrm{~V}$. The distribution of drift velocity $\left(\mathrm{V}_{\mathrm{x}}\right)$ and velocity component of electrons going forward $\left(\mathrm{V}_{\mathrm{fwd}}\right)$ are also shown in reference to BSC in Fig. 4.

Finally in Fig. 6, the scattering numbers and effective scattering numbers with and without impurity scattering are shown. The impurity scattering model is due to Brooks-Herring $(\mathrm{BH})$ and is stronger in the lower sheet electron density, so the case at $\mathrm{V}_{\mathrm{g}}=0.3 \mathrm{~V}$ is shown. Though the scattering number of $\mathrm{BH}$ is larger than that of phonons (Fig. 6(a)), the effective scattering number of $\mathrm{BH}$ is only $4.4 \%$ of corresponding scattering number thus much smaller than that of phonon (Fig. 6(b)).

Regarding $\mathrm{V}_{\mathrm{g}}$ dependence, the effective scattering number of total and surface scattering without $\mathrm{BH}$ at $\mathrm{V}_{\mathrm{g}}=0.3 \mathrm{~V}$ (Fig. 6(b)) is smaller than that at $\mathrm{V}_{\mathrm{g}}=0.9 \mathrm{~V}$ (Fig. 3 ), which is in good correspondence to the results of BSC (Fig. 4). 


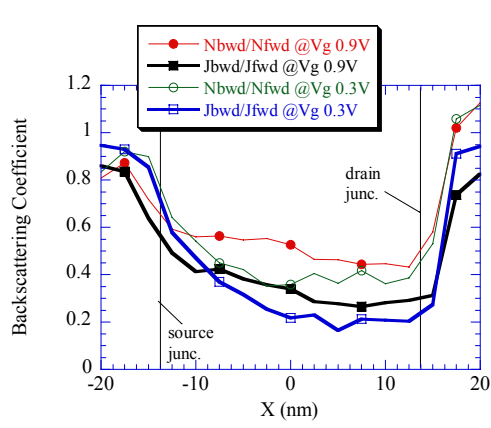

Figure 4: Back-scattering coefficients along the channel defined as 1) the ratio of number of electrons going forward to that going backward $\left(\mathrm{N}_{\text {bwd }} / \mathrm{N}_{\text {fwd }}\right)$, and 2) the ratio of current going forward to that going backward $\left(\left|\mathrm{J}_{\mathrm{bwd}} / \mathrm{J}_{\mathrm{fwd}}\right|\right)$. $\mathrm{V}_{\mathrm{d}}=0.9 \mathrm{~V}$.

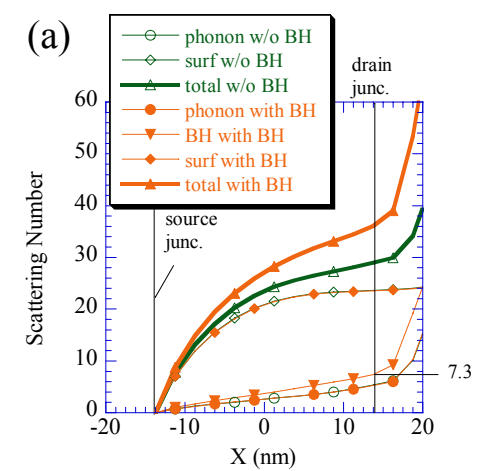

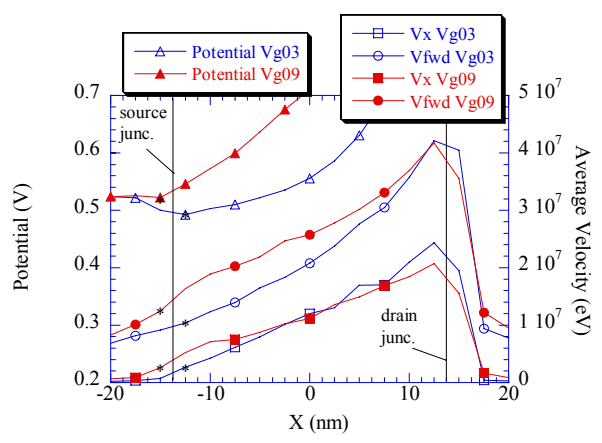

Figure 5: The distribution of potential, average drift velocity $\left(\mathrm{V}_{\mathrm{x}}\right)$ and average velocity component of electrons going forward $\left(\mathrm{V}_{\mathrm{fwd}}\right)$ along the channel. The asterisk $(*)$ indicate the position of the potential minima $\left(\psi_{\min }\right)$.

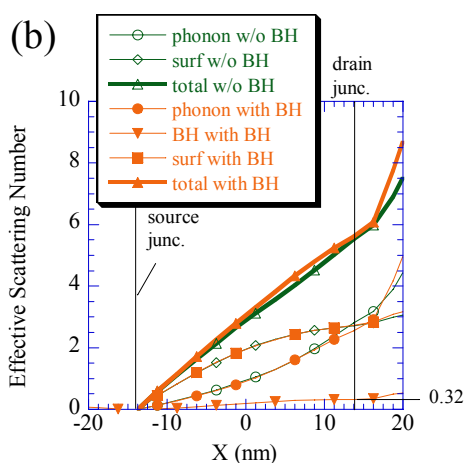

Figure 6: Cumulative (a) numbers and (b) effective numbers of scattering events along the channel with / without impurity scatterings $(\mathrm{BH}) . \mathrm{V}_{\mathrm{d}}=0.9 \mathrm{~V}, \mathrm{~V}_{\mathrm{g}}=0.3 \mathrm{~V}$.

\section{Conclusion}

The concept of 'effective' scattering number is proposed. This concept is shown to be useful to understand the quasi ballistic transport and the role of various scattering mechanisms especially when back-scattering is not negligible.

\section{References}

[1] K. Natori, J. Appl. Phys. 76, 4879 (1994).

[2] M. Lundstrom, IEEE Electron Dev. Lett., 18, 361 (1997).

[3] P. Palestri, et al., IEEE Trans. Electron Devices, 52, 2727 (2005).

[4] H. Tsuchiya, et al., IEEE Trans. Electron Devices, 53, 2965 (2006).

[5] Y. Ohkura, et al., SISPAD 2005, p. 279.

[6] Y. Ohkura, et al., SISPAD 2003, p. 67.

[7] Y. Park, et al., IEEE Trans. Electron Devices, 30, 1110 (1983).

[8] M. V. Fischetti and S. E. Laux, Phys. Rev. B, 38, 9721 (1988). 